\title{
A BURST OF STAR FORMATION IN GALAXIES OF THE M 81 GROUP
}

\author{
B.P. ARTAMONOV \\ Sternberg Astronomical Institute \\ University Av. 13 \\ 119899 Moscow B-234 \\ Russia
}

UBV photographic plates of galaxies (resolution 0"8) for the M 81 Group were obtained with the 1-metre Ritchey-Chretien (RC) Zeiss telescope at Maidanak Observatory. Some plates show a resolution $\sim 0$ 0.7. Artamonov et al. (1990) have distinguished and measured positions $( \pm 0.1)$ of starlike objects in the central regions of M 82 (69 spots) and NGC 3077 (19 spots). We have measured the position of 120 blue starlike objects in NGC 2976. Image processing was carried out using a PC 286/386 with calibration using the data of Notni \& Bronkalla, 1985; Bronkalla et al. 1992. The observed galaxies show active star formation which are related with intergalactic interaction. We have carried out UBV photometry of starlike objects in these galaxies. All objects emulate a population of young stars with different reddening. Figure 1 shows the twocolour plot of starlike objects in M 82 and the central regions of NGC 3077 (Price \& Gullixson 1989). The colours of these objects lie along the line of reddening.

After extinction corrections, the colours of the objects are found to lie in the region for Bstars. The objects in M 82 separate into two groups. The first group is found on the periphery of the central regions. The second group lies in the center of $M 82$ and has bluer colours. Spectral observation of starlike objects in these galaxies have confirmed the existence of two groups. The first group shows post-starburst spectra but the central group has spectra which are typical for strong bursts of star formation. Figure 2 shows the two-colour plot of blue objects of NGC 2976. Redder colours refer to the old population in the centre of the galaxy. The starlike objects in the edge of the Large Dust Cloud NGC 2976 (Bronkalla et al. 1992) have reddened colour. We have a mixture from old population and reddened young population but the two-colour plot shows a lot of blue objects like HII regions in the spiral arm.

The short conclusion is:

1) the middle ages of blue objects after correction for dust are in agreement with the simple evolution model (Artamonov 1993): viz. $10^{6}$ y $-\mathrm{HII}$ objects NGC $2976,10^{7}$ y - central objects M 82 and NGC $3077,5 \times 10^{7}-10^{8}$ y - peripheral disk objects M 82;

2) we note that the distribution of the objects is flatter when the group of objects is younger. The central group of SNR is flatter.

This research is supported by RFFR Grant $93 / 17060$. 


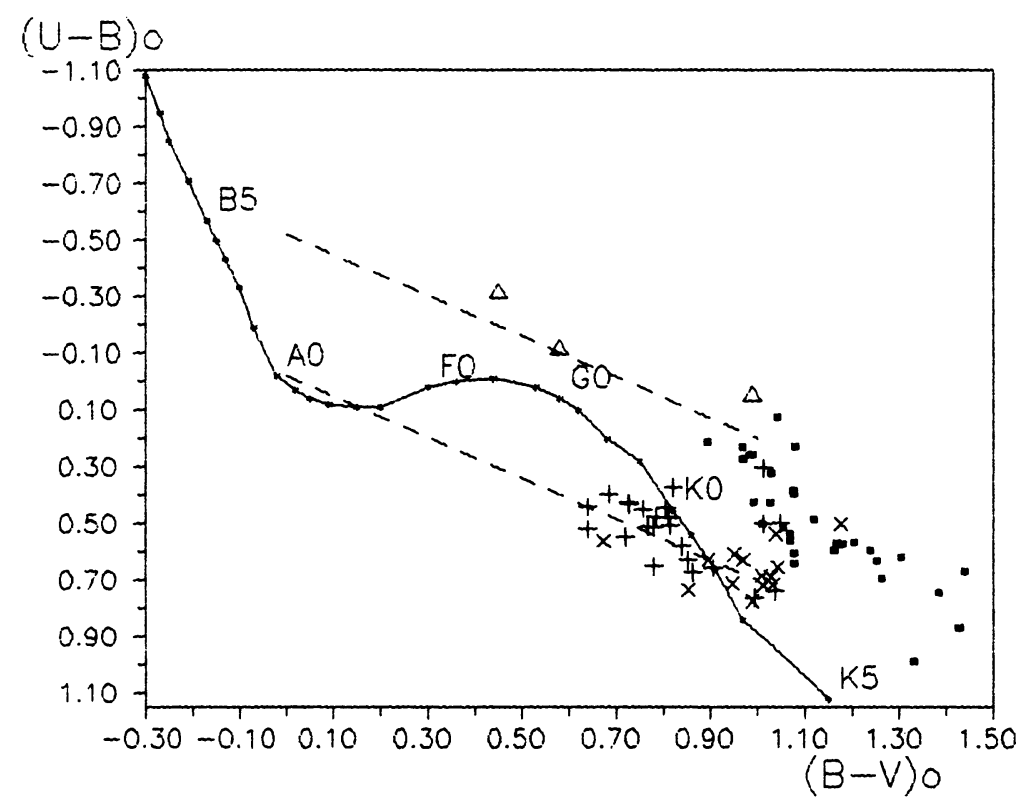

Figure 1. Two-colour plot for M 82 and NGC 3077. $\square$ - centre $M 82,+-$ EN region $M 82, x$ - WS region M 82, $\Delta$ - central regions NGC 3077 (Price \& Gullixson, 1989). The dashed line indicates the reddening.

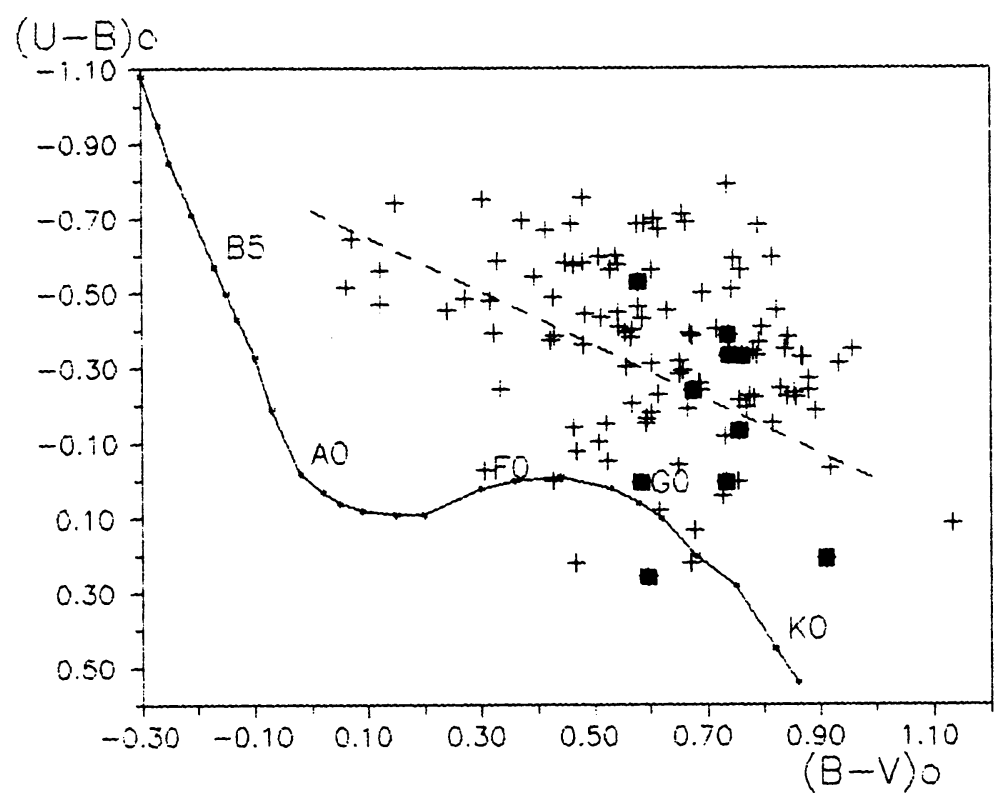

Figure 2. Two-colour plot of starlike objects in NGC 2976. - objects on the edge of Large Dust Cloud. The dashed line indicates the reddening. 


\section{References}

Artamonov, B.P., Novikov, S.B. and Shokin, Yu A., 1990. Preprint 16, Maidanak Obs. Sternberg Astron. Inst.

Artamonov, B.P., 1993. IAU Symp. 161. These proceedings.

Bronkalla, W., Notni, P. and Mutter, A.A.-R., 1992. Astron. Nachr., in press.

Notni, P. and Bronkalla, W., 1984. Astron. Nachr., 305, 157.

Price, J.S. and Gullixson, C.A., 1989. Astrophys. J., 337, 658. 